University of Nebraska - Lincoln

DigitalCommons@University of Nebraska - Lincoln

\title{
Survivable Virtual Topology Routing under Shared Risk Link Groups in WDM Networks
}

\author{
Ajay Todimala \\ University of Nebraska-Lincoln, ajayt@cse.unl.edu \\ Byrav Ramamurthy \\ University of Nebraska-Lincoln, bramamurthy2@unl.edu
}

Follow this and additional works at: https://digitalcommons.unl.edu/cseconfwork

Part of the Computer Sciences Commons

Todimala, Ajay and Ramamurthy, Byrav, "Survivable Virtual Topology Routing under Shared Risk Link Groups in WDM Networks" (2004). CSE Conference and Workshop Papers. 100.

https://digitalcommons.unl.edu/cseconfwork/100

This Article is brought to you for free and open access by the Computer Science and Engineering, Department of at DigitalCommons@University of Nebraska - Lincoln. It has been accepted for inclusion in CSE Conference and Workshop Papers by an authorized administrator of DigitalCommons@University of Nebraska - Lincoln. 


\title{
Survivable Virtual Topology Routing under Shared Risk Link Groups in WDM Networks *
}

\author{
Ajay Todimala and Byrav Ramamurthy \\ Department of Computer Science and Engineering \\ University of Nebraska-Lincoln \\ Lincoln NE 68588-0115 U.S.A. \\ Email: \{ajayt,byrav\}@cse.unl.edu
}

\begin{abstract}
Network survivability is one of the most important issues in the design of optical WDM networks. In this work we study the problem of survivable routing of a virtual topology on a physical topology with Shared Risk Link Groups (SRLG). The survivable virtual topology routing problem against single-link failures in the physical topology is proved to be NP-complete in [1]. We prove that survivable virtual topology routing problem against SRLG/node failures is also NP-complete. We present an improved integer linear programming (ILP) formulation (in comparison to [1]) for computing the survivable routing under SRLG/node failures. Using an ILP solver, we computed the survivable virtual topology routing against link and SRLG failures for small and medium sized networks efficiently. As even our improved ILP formulation becomes intractable for large networks, we present a congestion-based heuristic and a tabu search heuristic (which uses the congestion-based heuristic solution as the initial solution) for computing survivable routing of a virtual topology. Our experimental results show that tabu search heuristic coupled with the congestion based heuristic (used as initial solution) provides fast and near-optimal solutions.
\end{abstract}

\section{Introduction}

WDM networks have gained tremendous popularity due to their ability to tap the enormous amount of bandwidth in an optical fiber. Their growing popularity and bandwidth capacity have made survivability in these networks an important aspect. The physical topology of a WDM network consists of nodes interconnected with a pair of fiber

*This work was supported in part by the U. S. National Science Foundation grants (ANI-0074121 and EPS-0091900). links. The fact that fiber links pass through conduits, and right-of-way give rise to single-point failures. Such failures might result in failure of multiple links in the physical topology. In this work we study how to survivably route the virtual topology against such failure conditions. The virtual topology at the optical layer consists of a sub-set of nodes of the physical layer and an interconnection of logical links. Often the virtual topology is different from the physical topology and may need to be reconfigured with changing traffic patterns.

A Shared Risk Link Group (SRLG) is any sub-set of links present in the network that share the risk of failing at the same time. SRLG can be used to model several types of failure conditions like single-link failures, conduit/rightof-way failures, fiber-span failures, double-link failures or the failure of any other possible subset of links sharing a common risk. Such SRLG are called general SRLG. In this work we study single link failures, conduit/rightof-way SRLG and node-failures on the physical topology. Though we did not experiment with double-link failures, our work can be directly applied to double-link failures. Node failures are considered as a special case where all the links incident on the node fail. For a routing to be survivable under node failures the rest of the nodes should remain connected.

Given the physical topology and the traffic pattern the virtual topology design problem is to design a virtual topology considering throughput, delay, equipment cost and reconfigurability. The virtual topology design problem is known to be NP-hard [2], [3]. A survey of virtual topology design algorithms is presented in [4]. The virtual topology design problem was decomposed into four sub-problems [5], topology subproblem, virtual topology routing subproblem, wavelength assignment subproblem and traffic routing subproblem. The objective of the topology subproblem is to determine the optimum virtual topology to be imposed on the physical topology based 
on the the traffic demands. The virtual topology routing problem is to compute a physical path for each logical link in the virtual topology. The wavelength assignment subproblem deals with assigning a free wavelength along the computed physical path corresponding to each virtual link in the virtual topology considering the wavelength restrictions. The traffic routing subproblem is to compute a virtual path to route traffic between source and destination nodes in the virtual topology. The survivable routing problem studied in this paper can be used to solve the second and third sub-problems i.e., virtual topology routing subproblem and wavelength assignment subproblem assuming that the optimal virtual topology for the traffic pattern is already known. In order to survivably route traffic on the virtual topology, it needs to remain connected in the event of SRLG failures due to failure of single components like conduit, right-of-way, etc., in the physical topology. The routing of such a topology is called survivable virtual topology routing (SVTR).

The design of protected virtual topologies by minimizing the number of disconnected source destination pairs due to failure of single links in the physical topology was addressed in [6]. The SVTR problem against single link failures was addressed by Modiano et al., in [1]. The virtual topology design problem is well researched [4], [2], [3] but little work has been done on the survivability aspect of the virtual topology. The SVTR problem against single physical link failures was proved to be NP-complete in [1]. The work also presented the necessary conditions on the physical and virtual topologies for survivable routing to exist. The study then gave necessary and sufficient conditions for survivable routing and provided an ILP formulation. In [7] the survivable ring virtual topology routing problem is addressed. This work presented an algorithm to find the survivable routing if exists. In [8], [9] the survivable routing of logical topologies is considered. This work considers the addition of virtual links to make the virtual ring survivable. Paper [10] addressed the issue of designing protected multiple virtual private networks (VPN) on a single physical network. For each VPN a working and protection VPNs are designed considering single link and node failures. But neither of the working or protection VPN are themselves survivable. The problem of mapping a multi-graph Internet topology onto physical topology for survivability against link failures was addressed in [11].

In this work we study the survivable routing problem under SRLG or node failures. We provide an insight into the theory of cut-sets of a graph to present an improved (in comparison to [1]) necessary and sufficient conditions for a routing of virtual topology on the physical topology to be survivable. A cut-set of a graph is a set of edges, the removal of which disconnects the graph. A formal definition of cut-set is provided in Section 2. Based on the necessary and sufficient conditions, we present an ILP formulation for solving the SVTR problem. The rest of the paper is organized as follows. In section 2 we first give a classification of cut-sets of a graph and define the SVTR problem under SRLG. We also present improved necessary and sufficient conditions for survivable routing under SRLG or node failures based on the cut-set classification. In Section 3 we present the improved ILP formulation for survivable routing. As ILP formulations are difficult to solve for even medium-sized graphs, we present congestion-based heuristics for SVTR under single link failures in Section 4. In Section 5 we present the tabu search heuristic to solve the SVTR problem under SRLG. Section 6 presents the results from experiments of survivable routing considering different physical and virtual topologies. Section 7 provides the conclusions.

\section{Survivable Routing}

In this section we formalize the survivable virtual topology routing (SVTR) problem and present the necessary and sufficient conditions. We present the proof of correctness of the ILP formulation by proving the necessary and sufficient conditions for survivable routing of a virtual topology on the physical topology with SRLG. Let us discuss the problem of survivable virtual topology routing. The physical topology $G_{p}=\left(V_{p}, E_{p}\right)$ where $V_{p}$ is the set of nodes in the physical topology and $E_{p}$ is a set of bidirectional fiber links between nodes $(i, j) \in V_{p}$. A bidirectional fiber link is a pair of fiber links where each fiber is dedicated to carry data in a particular direction opposite to the other fiber. The SRLG for the physical topology $R_{p}=\left\{r_{i} \mid r_{i}=\left\{e_{i, 1}, e_{i, 2}, \ldots e_{i, m}\right\}, 1 \leq j \leq m,\left(e_{i, j} \in E_{p}\right.\right.$ and share the same risk of failure) $\}$. Each link in the physical topology belongs to at least one SRLG in the set $R_{p}$. This assumption is based on the fact that every physical link passes through some conduit (at least one of its own) and is a source of failure. When an SRLG $r \in R_{p}$ consists of all the links incident on a node $v \in V_{p}$, we consider it as the special case of node failure. The virtual or logical topology is a graph $G_{l}=\left(V_{l}, E_{l}\right)$ where $V_{l} \subseteq V_{p}$ and link $(i, j) \in E_{l}$ represents logical bi-directional link between nodes $i, j \in V_{l}$. Not all the nodes in the physical topology need to be present in the virtual topology. Some of the nodes are just tapping points [12]. A tapping point is a node in the network such that it is not a source or destination for any connection request.

For each link in the virtual topology, we need to find a path in the physical topology. Both, the fiber links in the physical topology and logical links in the virtual topology are bi-directional. We assume that both topologies are undirected and compute routing between the node pair 
assuming the direction is immaterial. The same path can be used to route the connection in both directions. (a)

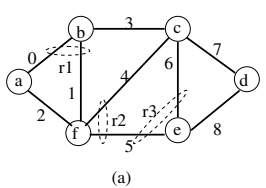

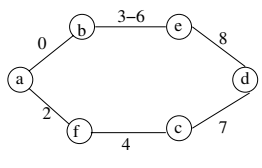

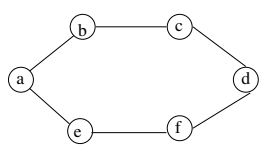

(c)

\begin{abstract}
Figure 1. (a). A 6-node physical topology with SRLG. (b). A survivable virtual topology and numbers adjacent to each virtual link show the routing. (c) A non-survivable virtual topology.
\end{abstract}

Given the physical topology $G_{p}$, the SRLG $R_{p}$ and the virtual topology $G_{l}$ we wish to determine a routing of the virtual topology such that in the event of failure of any single SRLG in the physical topology, the virtual topology is still connected. Such a routing is called SRLG survivable routing. In the rest of the document 'survivable' refers to 'SRLG survivable' unless specified explicitly. Let us illustrate the survivable virtual topology routing problem. Fig. 1(a) shows a physical topology with SRLG. Links $(a, b)$ and $(b, f)$ belong to the SRLG $r_{1}$ (indicated by dashed ovals). Similarly SRLG $r_{2}$ and $r_{3}$ containing two links each are shown in Fig. 1(a). Consider the virtual topology shown in Fig. 1(b). For each virtual link in the virtual topology there exists many different paths for routing on the physical topology. As shown in Fig. 1(b), the virtual link $(b, e)$ is routed on the physical links labeled 3 and 6 in the physical topology. Similarly, the physical route of other virtual links is shown in the Fig. 1(b). The routing is survivable against SRLG failures. If the link $(b, e)$ were routed on the links 1 and 5, then failure of SRLG $r_{1}$ will result in the failure of virtual links $(a, b)$ and $(b, e)$. Therefore the routing is not survivable. Survivable routing of a virtual topology exists if there exists atleast one routing of the virtual topology that is survivable. We call such a topology, survivable virtual topology. Now consider the virtual topology sown in Fig. 1(c). It is easy to see that any routing of the virtual topology Fig. 1(c) on the physical topology Fig. 1(a) is not survivable. Therefore survivable routing of the virtual topology in Fig. 1(c) does not exist. Such topology is called non-survivable virtual topology.

Let us introduce a few graph theoretic definitions and concepts from [13] before presenting the necessary and sufficient conditions for a survivable routing. Given an undirected connected graph $G=(V, E)$ where $V$ is the set of nodes and $E$ is the set of edges where $(i, j)$ represents edge between nodes $i$ and $j$. A cut $\langle S, V \backslash S\rangle$ is a partition of the set of nodes $V$ into two sub-sets $S$ and $V \backslash S . V \backslash S$ represents set $V$ minus set $S$. A cut-set $C S_{G}\langle S, V \backslash S\rangle$ associated with cut $\langle S, V \backslash S\rangle$ is defined as the set of edges in $E$ such that one end-point is in $S$ and the other is in $V \backslash S$.

$$
\begin{aligned}
& \underset{C}{C S_{G}}\langle S, V \backslash S\rangle=\{(i, j) \mid(i, j) \in E \wedge(i \in S) \wedge(j \in \\
& (V \backslash S))\}
\end{aligned}
$$

For any given undirected connected graph $G=(V, E)$ the number of possible cuts is $2^{n-1}$ which is exponential in terms of $n$ where $n$ is the number of nodes. The cut-set $C S_{G}\langle\{\}, V\rangle=\{\}$ is the trivial cut-set. The number of nontrivial cuts of $G$ is therefore given by $2^{n-1}-1$. An induced sub-graph of a graph $G=(V, E)$ by a sub-set of $V$ such that edge $(i, j)$ belongs to the sub-graph if and only if $(i, j)$ belongs to $G$. Let $G_{p}=\left(V_{p}, E_{p}\right)$ be the physical topology graph. $G_{p, i}=\left(V_{p, i}, E_{p, i}\right)$ is the induced sub-graph of $G_{p}$ by the node set $V_{p, i}$ where $V_{p, i}=V_{p}-\{i\}, i \in V_{p}$ and $E_{p, i}=\left\{(m, n) \mid(m, n) \in E_{p} \wedge((m \neq i) \wedge(n \neq i))\right\}$.

Now let us classify the cut-sets into primary and secondary based on the connectedness of the partitions $V$ and $V \backslash S$. A cut, $\langle S, V \backslash S\rangle$, is called a primary-cut if and only if both of the induced sub-graphs of $G$ by the node sets $S, V \backslash S$ are connected components. A cut, $\langle S, V S\rangle\rangle$, is called a secondary-cut if either of the induced sub-graphs of $G$ by the node sets $S, V \backslash S$ is not a connected component. The cutset associated with a primary-cut (secondary-cut) is called a primary cut-set (secondary cut-set). One of the important properties Lem. 2.1 of primary and secondary cut-sets is that every secondary cut-set is a union of disjoint primary cut-sets. This property is useful in proving the improved necessary and sufficient conditions of a survivable routing.

Lemma 2.1. Let $\langle S, V \backslash S\rangle$ be a secondary cut and without loss of generality let $X_{1}, X_{2}, \ldots, X_{m}$ be pairwise disjoint components of $S$ and there are no edges between them then $C S\langle S, V \backslash S\rangle=C S\left\langle X_{1}, V \backslash X_{1}\right\rangle \cup C S\left\langle X_{2}, V \backslash X_{2}\right\rangle \cup$ $\ldots C S\left\langle X_{m}, V \backslash X_{m}\right\rangle$.

\subsection{Necessary and Sufficient Conditions: SRLG Constraints}

Given an undirected physical topology $G_{p}$, its SRLG $R_{p}$ and a virtual topology $G_{l}$ where $V_{l} \subseteq V_{p}$. The virtual topology must be 2-edge-connected, otherwise the survivable routing does not exist. Every cut-set of the virtual topology then has at least 2 edges. If the virtual topology is only edge-connected then failure of some SRLG in the physical topology may result in failure of single edge in the cut-set, therefore it cannot be survivable. The necessary conditions on the physical topology for the existence of survivable routing is, due to the failure of any single SRLG the physical topology must not get disconnected. If the physical topology gets disconnected due to a single SRLG failure, then it is not possible to route the virtual topology such that it remains connected. 
Assuming that the physical and virtual topologies satisfy the necessary conditions for the existence of survivable routing, we state the necessary and sufficient conditions for a routing of $G_{l}$ on $G_{p}$ to be survivable. Theorem 2.2 gives the necessary and sufficient conditions for survivable routing of a virtual topology on a physical topology.

Theorem 2.2. Given the physical topology $G_{p}$, SRLG $R_{p}$ and the virtual topology $G_{l}$, the routing of $G_{l}$ over $G_{p}$ is said to be survivable if and only if $\forall r_{i} \in R_{p}$ and for all primary cut-sets $P C S_{G_{l}}\left\langle S, V_{l} \backslash S\right\rangle$ of the virtual topology at least one link in $P C S_{G_{l}}\left\langle S, V_{l} \backslash S\right\rangle$ is not routed over any of the links in $r_{i}=\left\{e_{i, 1}, e_{i, 2}, \ldots, e_{i, m}\right\}$.

Proof. Assume that the routing is survivable. Now suppose $\exists r_{i} \in R_{p}$ and a primary cut-set $P C S_{G_{l}}\langle S, V \backslash S\rangle$ such that all the links of the cut-set are routed over links in $r_{i}=\left\{e_{i, 1}, e_{i, 2}, \ldots, e_{i, m}\right\}$. Now failure of SRLG $r_{i}=$ $\left\{e_{i, 1}, e_{i, 2}, \ldots, e_{i, m}\right\}$ results in failure of all the links in the cut-set $P C S_{G_{l}}\langle S, V \backslash S\rangle$. So the virtual topology will get disconnected, contradicting the assumption that routing is survivable.

Now suppose that $\forall r_{i} \in R_{p}$ and for all primary cutsets $P C S_{G_{l}}\left\langle S, V_{l} \backslash S\right\rangle$ of the virtual topology at least one link in $P C S_{G_{l}}\left\langle S, V_{l} \backslash S\right\rangle$ is not routed over links in $r_{i}=$ $\left\{e_{i, 1}, e_{i, 2}, \ldots, e_{i, m}\right\}$. Therefore from the Lemma 2.1 of the secondary cut-sets which says, every secondary cut-set is the union of two or more disjoint primary cut-sets, it follows that there is at least one link in the secondary cut-set that is not routed on links $r_{i}=\left\{e_{i, 1}, e_{i, 2}, \ldots, e_{i, m}\right\}$. Hence failure of any single SRLG $\forall r_{i} \in R_{p}$ at least one link of all the non-trivial cut-sets (both primary and secondary) is not routed on the links in $r_{i}$. Therefore failure of $r_{i}$ results in a virtual topology which is still connected. Hence the routing is survivable.

Theorem 2.3. The survivable virtual topology routing (SVTR) problem under SRLG is NP-complete.

Proof. Consider a special case of the SVTR problem under SRLG where every single physical link belongs to its own SRLG i.e., $R_{p}=\left\{\left\{e_{i}\right\} \mid \forall e_{i} \in E_{p}\right\}$. This is the survivable routing problem under single link failure which was proved to be NP-complete [1]. So NP-completeness of survivability problem under SRLG follows.

\subsection{Necessary and Sufficient Conditions: Node Failures}

Given the undirected physical topology $G_{p}$ and undirected graph virtual topology $G_{l}$. The virtual topology is 2 vertex connected, otherwise the survivable routing does not exist. Let us first see the necessary conditions on the virtual topology for survivable routing of a virtual topology to exist, given by Theorem 2.4 .
Lemma 2.4. Given the physical topology $G_{p}$ and the virtual topology $G_{l}$. A routing of $G_{l}$ over $G_{p}$ exists if $\forall i \in V_{p}, G_{l, i}$ is connected.

Theorem 2.5 gives the necessary and sufficient for survivable routing of the virtual topology $G_{l}$ over the physical topology $G_{p}$ under single node failures.

Theorem 2.5. A routing is survivable if and only if for all $G_{l, i}$ for $i \in V_{p}$, at least one link in $P C S_{G_{l, i}}\left\langle S, V_{l, i}-S\right\rangle$ is not routed on edge set $\left(E_{p}-E_{p, i}\right)$ where $E_{p, i}$ is the edge set of induced sub-graph of $G_{p}, G_{p, i}=\left(V_{p, i}, E_{p, i}\right)$.

Proof. Suppose that routing is survivable. Now suppose for contradiction that there exists $i \in V_{p}$ such that all the links of cut-set $P C S_{G_{l, i}}\left\langle S, V_{l, i}-S\right\rangle$ are routed on the edge set $\left(E_{p}-E_{p, i}\right)$. Now if node $i$ fails in $G_{p}$, all the links in the links in the cut-set $P C S_{G_{l, i}}\left\langle S, V_{l, i}-S\right\rangle$. So the remaining nodes in the virtual topology $G_{l, i}$ gets disconnected. A contradiction.

Now suppose that for all $G_{l, i}$ for $i \in V_{p}$, at least one link in $P C S_{G_{l, i}}\left\langle S, V_{l, i}-S\right\rangle$ is not routed on edge set $\left(E_{p}\right.$ $\left.E_{p, i}\right)$. Therefore from the Lemma 2.1 of the secondary cutsets which says, every secondary cut-set is the union of two or more disjoint primary cut-sets, it follows that there is at least one link in the secondary cut-set that is not routed on links $\left(E_{p}-E_{p, i}\right)$. Hence failure of any node $i \in V_{p}$ at least one link of all the non-trivial cut-sets (both primary and secondary) is not routed on the links in $\left(E_{p}-E_{p, i}\right)$. Therefore failure of $i$ results in a virtual topology $G_{l, i}$ which is still connected. Hence the routing is survivable.

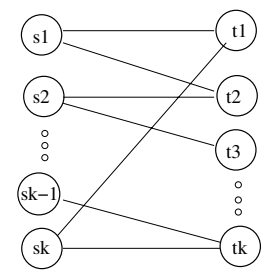

Figure 2. The virtual topology network.

Theorem 2.6. Survivable virtual topology routing (SVTR) problem under node failures is NP-complete.

Proof. SVTR $\in N P$. Given the $\left\langle G_{p}, G_{l}\right\rangle$ and the routing $R$ of $G_{l}$ over $G_{p}$, it can be verified if the routing is survivable. A routing $R$ is defined as a mapping from set of links in $G_{l}$ to set of paths in $G_{p}$. For each node $i \in V_{p}$ compute $G_{p, i}$ the induced graph of $G_{p}$ by $V_{p} \backslash\{i\}$. Given the routing $R$ compute the corresponding $G_{l, i}^{\prime}$ by removing all the link in $G_{l, i}$ that are routed through the node $i$, where $G_{l, i}$ is the induced sub-graph of $G_{l}$ by $V_{l} \backslash\{i\}$. Check if $G_{l, i}^{\prime}$ is 
connected by depth first search algorithm. This can be done in $O(V+E)$. So the survivable routing can be checked in $O(V(V+E))$.

To show survivable routing is NP-hard we give a reduction from Disjoint Connecting Paths Problem (DCPP) [14] to SRP. DCPP is given an undirected graph $G=$ $(V, E)$, and a collection of $k$ disjoint vertex pairs $P=$ $\left(s_{1}, t_{1}\right),\left(s_{2}, t_{2}\right), \ldots\left(s_{k}, t_{k}\right)$, does $G$ contain $k$ mutually vertex-disjoint paths, one connecting $s_{i}$ and $t_{i}$ for each $\mathrm{i}$, $1 \leq i \leq k$. DCPP is known to be NP-complete. Given $\langle G, P\rangle$, the reduction computes $\left\langle G_{p}, G_{l}\right\rangle$. Compute $G_{p}=$ $\left(V_{p}, R_{p}\right)$ where $V_{p}=V$ and $E_{p}=E \cup\left\{\left(s_{i}, t_{i+1}\right) \mid 1 \leq i<\right.$ $k)\} \cup\left\{\left(s_{k}, t_{1}\right)\right\} . G_{l}$ is a cycle $s_{1} t_{2} s_{2} t_{3} s_{3} \ldots s_{k-1} t_{k} s_{k} t_{1} s_{1}$. The virtual topology is shown in Fig. 2.

Claim: $\langle G, P\rangle$ has $k$ mutually vertex-disjoint paths if and only if $\left\langle G_{p}, G_{l}\right\rangle$ has survivable routing.

Suppose that $\langle G, P\rangle$ has $k$-vertex disjoint paths. Implies that paths $s_{i}$ to $t_{i}, 1 \leq i \leq k$ are vertex disjoint in $G$. Implies that they are also vertex disjoint in $G_{p}$. It implies that none of the paths use the links $\left(s_{1}, t_{2}\right),\left(s_{2}, t_{3}\right), \ldots\left(s_{k-1}, t_{k}\right),\left(s_{k}, t_{1}\right)$. Now consider the following routing: for each $i, 1 \leq i \leq k,\left(s_{i}, t_{i}\right)$ is routed on the vertex disjoint path in $G_{p}$. And the remaining logical links in $\left.G_{l},\left\{\left(s_{i}, t_{i+1}\right) \mid 1 \leq i<k\right)\right\} \cup\left\{\left(s_{k}, t_{1}\right)\right\}$ are routed on the corresponding links $\left\{\left(s_{i}, t_{i+1}\right) \mid 1 \leq i<\right.$ $k)\} \cup\left\{\left(s_{k}, t_{1}\right)\right\}$ in $G_{p}$. This routing is survivable.

Now assume that the survivable routing of $\left\langle G_{p}, G_{l}\right\rangle$ exists. Suppose for contradiction that vertex disjoint paths does not exist in $G$ for the following node pair $\left(s_{i}, t_{i}\right)$ and $\left(s_{j}, t_{j}\right)$. It implies that routing $R$ of links $\left(s_{i}, t_{i}\right)$ and $\left(s_{j}, t_{j}\right)$ in $G_{p}$ is also not vertex disjoint. Let $x \in V_{p}$ be the vertex in common through which both the link $\left(s_{i}, t_{i}\right)$ and $\left(s_{j}, t_{j}\right)$ are routed. Now failure of this node $x$ will result in disconnected logical topology $G_{l, x}^{\prime}$, a contradiction. Therefore $\langle G, P\rangle$ has $k$-vertex disjoint paths.

\section{Integer Linear Programming Formulation}

Given the undirected physical topology $G_{p}=\left(V_{p}, E_{p}\right)$, the SRLG $R_{p}$ and the virtual topology $G_{l}=\left(V_{l}, E_{l}\right)$. Let us assume that both $G_{p}$ and $G_{l}$ satisfy the necessary conditions for the existence of survivable routing. For each virtual link $(s, t)$ in the virtual topology, we need to compute a path in the physical topology. Let $f_{i j}^{s t}=1$ if the virtual link between $(s, t)$ is routed over the physical link $(i, j)$ and 0 otherwise. The formulation below follows that in [1] by enhancing the survivability constraint in the formulation and generalizing it to SRLG networks.

The objective of the ILP formulation is to minimize the total number of wavelength links used for survivable virtual topology routing. The formulation follows:
Minimize

$$
\sum_{\substack{(i, j) \in E_{p} \\(s, t) \in E_{l}}} f_{i j}^{s t}
$$
$V_{p}$

Subject to connectivity constraints: $\forall(s, t) \in E_{l}, \forall i \in$

$\sum_{j s . t .(i, j) \in E_{p}} f_{i j}^{s t}-\sum_{j s . t .(j, i) \in E_{p}} f_{j i}^{s t}=\left\{\begin{aligned} 1, & \text { if } \mathrm{s}=\mathrm{i} \\ -1, & \text { if } \mathrm{t}=\mathrm{i} \\ 0, & \text { otherwise }\end{aligned}\right.$

SRLG survivability constraints: $\forall r \in R_{p}$ and $\forall$ primary cut-set $\left\langle S, V_{l} \backslash S\right\rangle$

$$
\sum_{(s, t) \in\left\langle S, V_{l} \backslash S\right\rangle,(i, j) \in r} f_{i j}^{s t}+f_{j i}^{s t}<\left|\left\langle S, V_{l} \backslash S\right\rangle\right| .
$$

Node survivability constraints: $\forall G_{l, i} i \in V_{p}$ and $\forall P C S_{G_{l, i}}\left\langle S, V_{l, i}-S\right\rangle$

$$
\sum_{\substack{(s, t) \in P C S\left\langle S, V_{l}-S\right\rangle \\(i, j) \in E_{p}-E p, i}} f_{i j}^{s t}+f_{j i}^{s t}<\left|P C S\left\langle S, V_{l, i}-S\right\rangle\right| .
$$

Wavelength assignment constraints: $\forall(s, t) \in E_{l}$

$$
\sum_{1 \leq x \leq W} \lambda^{s t}(x)=1
$$
$W$

Wavelength usage constraints: $\forall(i, j) \in E_{p}, 1 \leq x \leq$

$$
\sum_{(s, t) \in E_{l}} \lambda_{i j}^{s t}(x)+\lambda_{j i}^{s t}(x) \leq 1
$$

Capacity constraints: $\forall(i, j) \in E_{p}$

$$
\sum_{(s, t) \in E_{l}} f_{i j}^{s t}+f_{j i}^{s t} \leq W
$$

Constraints on $\lambda_{i j}^{s t}(x): \forall(s, t) \in E_{l}, \forall(i, j) \in E_{p}, x \in$ $\{1 \ldots W\}$

$$
\begin{gathered}
\lambda_{i j}^{s t}(x)+\lambda_{j i}^{s t}(x) \leq \lambda^{s t}(x) \\
\lambda_{i j}^{s t}(x)+\lambda_{j i}^{s t}(x) \geq \lambda^{s t}(x)+f_{i j}^{s t}+f_{j i}^{s t}-1 \\
\lambda_{i j}^{s t}(x)+\lambda_{j i}^{s t}(x) \leq f_{i j}^{s t}+f_{j i}^{s t}
\end{gathered}
$$

Integer constraints:

$$
f_{i j}^{s t} \in\{0,1\}
$$


The connectivity is modeled as a multi-commodity flow. Equation 2 gives the connectivity constraints of routing one unit of flow from node $s$ to $d$. Equation 3 gives the survivability constraints under single SRLG failures which is a direct consequence of Theorem 2.2. Only primary cut-sets of the virtual topology are considered instead of all cut-sets. This reduces the number of constraints and results in improved performance in terms of time. Equation 4 gives the survivability constraint against single node failures which is a direct consequence of Theorem 2.5. It is important to note that the above formulation gives the solution with the same minimum objective value as the formulation in [1]. $\quad \lambda^{s t}(w)=1$ if wavelength $w$ is assigned on along the physical route for the virtual link $(s, t)$ otherwise 0 . Constraint 5 states that one and only wavelength is assigned to physical path corresponding to every logical link in the virtual topology. $\lambda_{i j}^{s t}(w)=1$ if wavelength $w$ is assigned to link $(i, j)$ in physical topology for routing the virtual link $(s, t)$ otherwise 0 . Since no wave conversion is assumed wavelength continuity constraint is followed. Constraint 6 states that a wavelength can be used at most once on any given link in the physical topology. The capacity constraint 7 states that the total number of logical links that physical link $(i, j)$ can support is at most $W$. Constraint 8 states that if wavelength $x$ is not assigned to the logical link between nodes $s$ and $d$ then wavelength $x$ is not assigned on any physical link for routing this logical link. Constraint 9 states that if logical link $(s, t)$ is routed on physical link $(i, j)$ or $(j, i)$ and $w$ is the wavelength assigned to the logical link $(s, t)$ then $w$ wavelength is reserved along the $(i, j)$ or $(j, i)$ respectively for logical link $(s, t)$. Constraint 10 states that wavelength $x$ is reserved along link $(i, j)$ or $(j, i)$ for logical link $(s, t)$ if the logical link $(s, t)$ is routed along the link $(i, j)$ or $(j, i)$ respectively. Constraint 11 states that all the variable are binary variables.

\section{Congestion-Based Heuristics}

The ILP formulation can find optimal solutions for small networks but it takes exponential time with increasing input size. In this section we explore congestion-based heuristics for survivably routing a virtual topology on a physical topology. We already know that it is not possible to compute survivable routing under SRLG in polynomial time. Therefore, we use the approximation of minimizing the number of logical links that are routed on any given physical link i.e., minimizing congestion on a physical link. The intuition is to minimize the number of logical link failures due to the failure of any single physical link. It is important to note that these heuristics do not guarantee the survivable routing of a virtual topology but give fast and easy approximate solutions. We use this heuristic solution as an initial solution for the tabu search procedure to be discussed in the next section.

Given a physical topology $G_{p}=\left(V_{p}, E_{p}\right)$ and a simple at least 2-connected virtual topology $G_{l}=\left(V_{l}, E_{l}\right)$. Let us assume that both the topologies are undirected. In fact all physical and logical links are bi-directional. We only compute the route between the node pair $(s, t) \in$ $E_{l}$. For each virtual link $(s, t) \in E_{l}$ a set of $m$ shortest candidate paths $P_{s t}=\left\{p_{s t}^{1}, p_{s t}^{2}, \ldots p_{s t}^{m}\right\}$ are pre-computed [15]. This heuristic addresses the problem by dividing the problem into two sub-problems. The first sub-problem is to determine a strategy to select a virtual link to be routed next among the virtual links that are yet to be routed. The second sub-problem is to determine a strategy for selecting a path on the physical topology for routing the logical link among the $m$ pre-computed candidate paths.

For addressing the first sub-problem of selecting a virtual link to be routed next, there may exist many different strategies but we choose to experiment with the shortest path (S). With the shortest path strategy, virtual links are ordered in increasing order of the shortest path lengths in the physical topology. Now let us discuss the heuristics used to select a path from among the $m$ candidate shortest paths. There may exist many different strategies but we choose to explore the following: least congested path (LCP), and least congested average path (LCAP). With LCP strategy, a least congested path is chosen from among the candidate paths. Congestion of a path is defined as the congestion of the most congested link in the network. Congestion of a link is defined as the number of used wavelengths on the link. Average congestion of a path is defined as the average of congestion on all the link along the path. LCAP is same as LCP strategy except that if multiple least congested paths exist a path with least average congestion is chosen.

\section{Tabu Search Heuristic}

Tabu search is a meta heuristic that guides the search process of hard optimization problems. We give a general description of the tabu search process and then present the design of a simple tabu search heuristic for solving the SVTR problem. Given the problem description $P$, its initial solution $S_{i n i}$ and the objective function $\operatorname{Obj}(S)$, the tabu heuristic searches the solution space $S S$ of the problem for the optimal or close-to-optimal solution $S_{\text {opt }}$. The solution $S$ is a function from set of virtual links $(s, t)$ in the virtual topology to an index into the pre-computed path set of the corresponding node pair $(s, t)$ in the physical topology. This function $S$ is called routing of the virtual topology over the physical topology. The transformation or move from one solution $S_{s t}^{i}$ to another solution $S_{s t}^{j}$ in the solution space $S S$ is defined as changing the mapping of a virtual link $(s, t)$ from path $p_{s t}^{i}$ to $p_{s t}^{j}$ in its corresponding pre-computed path set $P_{s t}$. As already stated, for each 
virtual link $(s, t) \in E_{l}$ a set of $m$ shortest candidate paths $P_{s t}=\left\{p_{s t}^{1}, p_{s t}^{2}, \ldots p_{s t}^{m}\right\}$ is pre-computed. Neighborhood of a solution $S, N(S)$, is defined as solution space obtained by a single move from $S$. During the tabu search process of moving from one solution to another in its neighborhood, it records the best solution found so far. A move from $S_{s t}^{i}$ to $S_{s t}^{j}$ is said to be an improving (non-improving) move if and only if $\operatorname{Obj}\left(S_{s t}^{j}\right)$ is less than (greater than equal to) $\operatorname{Obj}\left(S_{s t}^{i}\right)$. The tabu search process is an interleaved sequence of improving and non-improving moves. The outline of the tabu search procedure is described below.
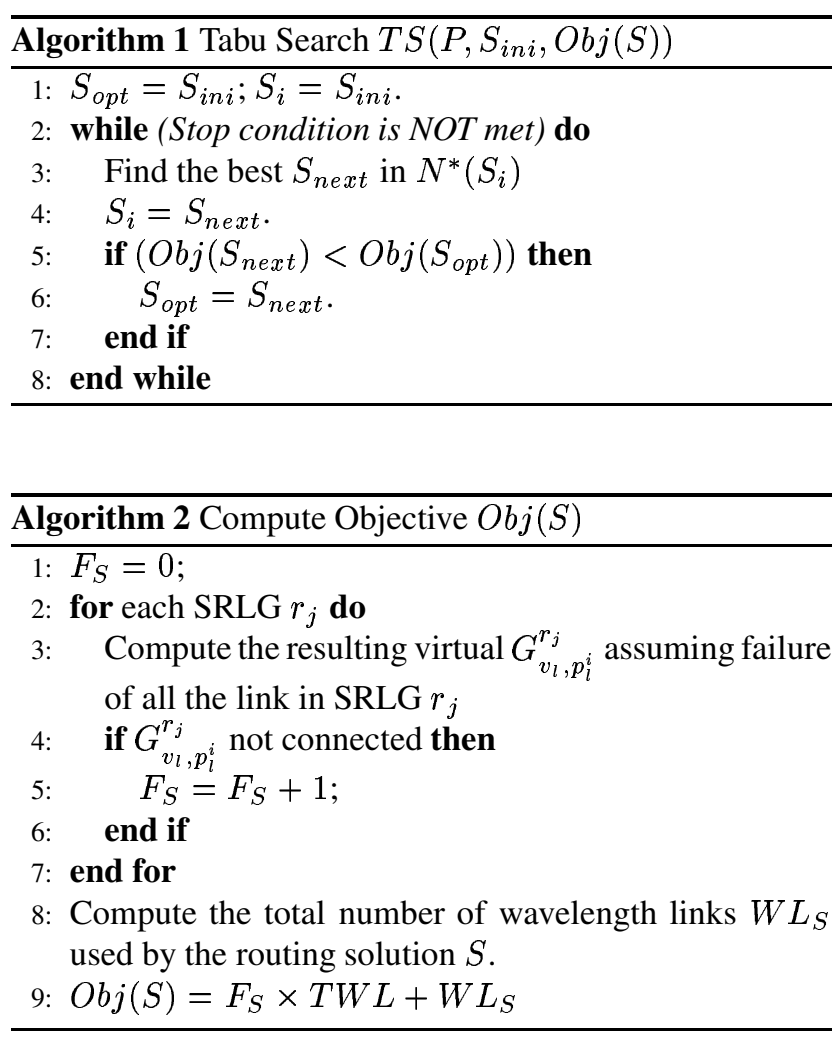

The stop condition for the tabu search may be one or more of the following: 1. A predefined number of total moves. 2. A predefined number of non-improving moves since the last improving move. and 3. If $N^{*}\left(S_{i}\right)$ is empty. The search stops if either of the above conditions is satisfied. The solution provided by the above congestion based heuristics is used as the initial solution to the tabu search process. The congestion based heuristics do not guarantee that the routing solution provided is survivable though survivability is a constraint of the SVTR problem. We also know that the objective of the SVTR problem is to minimize the number of wavelength links used for survivable routing. So the tabu search procedure has to search for a solution which is not only survivable but also optimal or close to optimal in terms of number of wavelength links used for routing. Therefore it has two objectives. The first objective is to minimize the number of component failures in the physical topology for which virtual topology gets disconnected. The second objective is to minimize the number of wavelength links used for routing. Minimizing the second objective, minimizes the cost of the network. As survivability is the constraint of our survivable routing problem, the first objective is given priority over the second objective. The objective function of the routing solution $S$ is defined as follows: $\operatorname{Obj}(S)=$ $F_{S} \times T W L+W L_{S}$, where $F_{S}$ is the number of SRLG failures in the physical topology for which virtual topology gets disconnected. $T W L$ is the total number of wavelength links in the physical topology, $T W L=W \times L$ where $W$ is the number of wavelengths on a fiber link and $L$ is the number of links in the physical topology. $W L_{S}$ is the number of wavelength links used by the routing solution $S$.

For a given solution the number of neighboring solutions are $O\left(E_{2} . m\right)$ where $E_{2}$ is the number of links in the virtual topology and $m$ is the maximum number of precomputed paths. Since for each move there are $E_{2}$ possible choices for selecting a virtual link and $m-1$ choices for selecting a candidate path for changing the mapping of the virtual link. For the transformation in a single iteration, the best neighboring solution (i.e., solution with minimum objective) need to be determined among all the neighboring solutions. In fact, in our tabu search procedure not the all neighboring solutions are examined for computing the best solution. A tabu tenure $t$ is assigned to each path selected for the virtual link during move of each iteration. The same path is not considered until the tabu tenure expires. Given a routing solution the procedure 2 computes the objective function $\operatorname{Obj}(S)$. The complexity of step 3 in Algorithm 2 is $O\left(E_{2} . P\right)$ and it is executed $C$ times where $P$ is the maximum number of links in an SRLG and $C$ is the number of SRLG. Therefore the worst case complexity of one iteration of the tabu search is $O\left(E_{1}^{2} \cdot m . C . P\right)$.

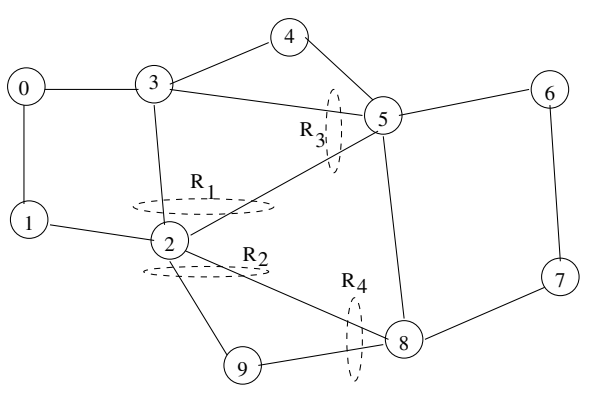

Figure 3. The 10-node Physical Net1. 


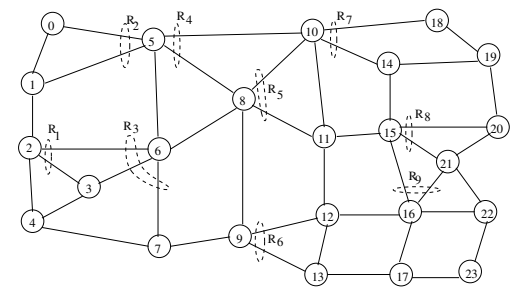

Figure 4. The 24-node Physical Net2.

\section{Experimental Design and Results}

Extensive simulations were conducted on 10-node, 11node NJLATA, and 24-node networks labeled as Net1, NJLATA network, and Net2 respectively (with increasing number of nodes). The physical topologies Net1 and Net2 are shown in Figures 3 and 4 respectively. The Net1 has 15 links and 13 SRLG. Four SRLG containing more than one link (two links) are shown in the Fig. 3. The remaining 9 links belong to SRLG of its own and are not shown in the Fig. 3. In our experiments we used randomly generated degree- $k$ regular graphs and $m$-link general graphs as virtual topologies. An undirected graph is said to be degree- $k$ regular if all the nodes in the graph have exactly degree $k$. We make sure that the randomly generated graphs satisfy the necessary conditions for the existence of survivable routing.

Table 1. Acronym table

\begin{tabular}{|r|l|}
\hline Acronym & Word \\
\hline \hline$V T$ & Virtual Topology Type \\
\hline$P T$ & Physical Topology \\
\hline No. $W a$ & Number of Wavelengths \\
\hline No. $V T$ & No. of Virtual Topologies \\
\hline No. $N S V T$ & No. of Non-Survivable Virtual Topologies \\
\hline No. UPVT & No. of Un-Protected Virtual Topologies \\
\hline$A v g . T i$ & Average Time (in sec) \\
\hline$A v g . S T i$ & Average Survivable Time (in sec) \\
\hline No. $B N W$ & $\begin{array}{l}\text { No. of Blocked topologies due to } \\
\text { No-free Wavelength }\end{array}$ \\
\hline No. Cons & No. of Constraints \\
\hline
\end{tabular}

We implemented and simulated our new ILP formulation (NEW-ILP) and ILP formulation presented in [1] (OLDILP). We also simulated congestion based heuristic (SLCAP) and the tabu search (TBS) heuristic. The ILP formulations are solved using ILOG CPLEX software package. The simulation experiments for Network1, NJLATA and Network2 are run on Sun Sparc Ultra60 workstation and the simulations for Net2 are run on
1.13 GHz AMD Athlon with 512 MB RAM. The ILP formulations were used for computing survivable routing only on Network1, NJLATA and Network2 but not on Network3 due to exponential growth in problem size. The performance of the ILP formulations and heuristics is measured in terms of number of wavelength links used for survivable routing and time taken to find the routing. Table 1 lists the acronyms used in the rest of the paper. As stated earlier two types of virtual topologies are used degree- $k$ regular and $m$-link general graphs, $V T T$ specifies the type of virtual topology. The acronym No. Wa gives the number of wavelengths on a fiber link in the physical topology. We assume that all the fiber links have same number of wavelengths. The acronym No. VT gives the number of virtual topologies simulated in a single experiment. The acronym No. NSVT gives the number of topologies for which survivable routing does not exist. The acronym No. UPVT represents the number of topologies for which routing computed was not survivable, including the topologies for which survivable routing does not exist. The acronym Avg. Ti represents the time taken to compute the survivable routing in seconds averaged over No. VT. The acronym No. SS represents the number of susceptible SRLGs of the No. UPVT of the heuristics. The acronym Avg. $S T$ gives the time taken by the tabu search to compute the survivable routing averaged over the number of survivable routings computed by tabu search. The acronym No. $B N W$ represents the number of blocked survivable routings due to no-free wavelength.

\section{Table 2. Survivable routing of virtual topologies on Net1 and NJLATA using ILP formulations.}

\begin{tabular}{|c|c|c|c|c|c|}
\hline$I L P$ & $P T$ & $V T T$ & $\begin{array}{c}\text { No. } \\
V T\end{array}$ & $\begin{array}{c}\text { No. } \\
\text { NSVT }\end{array}$ & $\begin{array}{c}\text { Avg. Ti } \\
\text { (in sec) }\end{array}$ \\
\hline \hline OLD & Net1 & degree-3 & 500 & 0 & 8.49 \\
\hline NEW & Net1 & degree-3 & 500 & 0 & 3.23 \\
\hline OLD & Net1 & 15 -link & 300 & 18 & 15.22 \\
\hline NEW & Net1 & 15 -link & 300 & 18 & 4.43 \\
\hline OLD & NJLATA & 20 -link & 800 & 0 & 26.2 \\
\hline NEW & NJLATA & 20 -link & 800 & 0 & 9.53 \\
\hline
\end{tabular}

Table 2 presents the results of survivable routing of degree-3 and 15-link virtual topologies on Net1 and 20-link virtual topologies on NJLATA using the ILP formulations NEW-ILP and OLD-ILP. We assume that number of wavelengths available for physical topologies Net1 and NJLATA are 4 and 6 respectively. The survivable routing existed for all the 500 virtual topologies Net1. Among 300 15-link virtual topologies survivable roting does not exist 
for 18 topologies. The average time taken for computing survivable routing by NEW-ILP are less than that of OLDILP. The average optimal number of wavelength links used for survivable routing is 30.17 for degree-3 and 30.57 for 15 -link virtual topologies. For all the 800 20-link virtual topologies survivable routing existed on NJLATA and the average optimal number of wavelength links used is 35.13 . Table 3 shows the number of survivability constraints for both of the ILP formulation (OLD-ILP) and (NEW-ILP) for various physical and virtual topologies. The physical topology EURO-NET is a 19 node network.

\section{Table 3. Number of constrains of ILP formulations for different physical and virtual topologies.}

\begin{tabular}{|c|c|c|c|}
\hline ILP & PT & VTT & No. Cons \\
\hline \hline NEW & EURO-NET & degree-3 & 32665 \\
\hline OLD & EURO-NET & degree-3 & 10223600 \\
\hline NEW & Net1 & degree-3 & 2830 \\
\hline OLD & Net1 & degree-3 & 9709 \\
\hline NEW & NJLATA & 20-link & 6083 \\
\hline OLD & NJLATA & 20-link & 18414 \\
\hline
\end{tabular}

Table 4. Surviable routing of virtual topologies on Net1 using TBS heuristic.

\begin{tabular}{|c|c|c|c|c|}
\hline VTT & No. Wa & No. UPVT & No. BNW & Avg. ST \\
\hline \hline degree-3 & 4 & 0 & 42 & 0.148 \\
\hline degree-3 & 6 & 0 & 0 & 0.148 \\
\hline 15-link & 4 & 30 & 57 & 0.369 \\
\hline
\end{tabular}

Table 5. Survivable routing of virtual topologies on Net2 using TBS heuristic.

\begin{tabular}{|c|c|c|c|c|}
\hline VTT & No. Wa & No. UPVT & No. BNW & Avg. ST \\
\hline \hline degree-3 & 8 & 16 & 56 & 7.16 \\
\hline 40-link & 8 & 103 & 136 & 7.25 \\
\hline 45-link & 8 & 18 & 280 & 3.45 \\
\hline 45-link & 12 & 18 & 13 & 3.45 \\
\hline
\end{tabular}

Table 4 presents the results of computing the survivable routing of 500 and 300 degree-3 and 15-link virtual topologies respectively on Net1 physical topology using TBS heuristic (with congestion-based heuristic solution as initial solution). The maximum number of $m$ of precomputed paths for each node pair is 15. TBS found the survivable routing for all the degree-3 topologies but failed to find survivable routing for 30 of 300 15-link virtual topologies. It is important to note here that out of 30 topologies survivable routing did not exist for 18 topologies. When 4 wavelengths are used 42 of the degree3 topologies were blocked and none were blocked when 6 wavelengths were used. Approximate time for computing the survivable routing for a virtual topology is measured as $6.4 \mathrm{sec}$. The average number of wavelength links used for survivable routing of degree-3 and 15-link virtual topologies are 30.34 and 31.1 respectively (very close to optimal value computing using ILP of 30.17 and 30.57 respectively).

Table 5 gives the results of computing survivable routing for 600 of degree-3, 40-link and 45-link virtual topologies on Net 2 using TBS heuristic with congestionbased heuristic solution as initial solution. The maximum number $m$ of pre-computed paths used for every node pair is 20. As ILP formulations could not be solved for the 24node network, it could not be verified if survivable routing existed for all the 600 degree- 3 randomly generated virtual topologies. TBS heuristic computed survivable routing for $97.3 \%$ of the degree-3 virtual topologies assuming survivable routing existed for all the virtual topologies. Among the 16 topologies for which survivable routing could not be computed, on average 1.3 SRLGs were vulnerable. The average numberof wavelength links used for computing survivable routing of degree-3, 40-link and 45-link virtual topologies are $111.65,123.57$ and 137.32 respectively. When considering $\mathrm{m}$-link arbitary virtual topologies, the number of un-protected virtual topologies are 11 times more than the number of un-protected topologies in degree-3 regular networks. When the number of links in the virtual topology is increased to 45 , the number of un-protected topologies decreses to as low as $2 \%$. In our experiments we observe that regular topologies are more survivable than arbitary topologies.

\section{Conclusions}

In this work we study the problem of survivably routing a virtual topology on a physical topology under SRLG and node failures. We presented an improved ILP formulation to solve the problem. As a proof of correctness of the ILP formulation, we proved the necessary and sufficient conditions for a routing of virtual topology on the physical topology under SRLG/node failures to be survivable. The ILP formulations were used to solve survivable routing for 10-node, and 11-node NJLATA, on degree-3 regular and arbitrary $m$-link general graphs as virtual networks. The improved ILP formulation enables to find the survivable routing 3-18 times faster in seconds in comparison to the formulation presented in [1]. The advantage of the speedup 
in time is that it increases the size of the topologies for which ILP can be used to compute the survivable topology routing problem. For large sized networks, we presented a tabu search heuristic. For computing the initial solution for the tabu search heuristic, we presented a congestion-based heuristic. We have simulated tabu search heuristic on 10node, and 11-node NJLATA in addition to 24-node network as physical networks. The experimantal results suggest that the tabu search heuristic computed the survivable routing for almost all the virtual topologies under SRLG while at the same time using close to optimal wavelength-links for routing. In case where survivable routing could not be found it greatly decreased the number of susceptible SRLGs for which the virtual topology gets disconnected.

\section{References}

[1] E. Modiano and A.Narula-Tam, "Survivable Lightpath Routing: A New Approach to the design of WDMBased Networks," IEEE Journal on Selected Areas in Communications, vol. 20, no. 4, pp. 800-809, May 2002.

[2] D. Banerjee and B. Mukherjee, "A practical approach for routing and wavelength assignment in large wavelengthrouted optical networks," IEEE Journal of Selected Areas in Communications vol. 14, no. 5, pp. 903-908, June 1996.

[3] R. Ramaswami and K. N. Sivarajan, "Design of logical topologies for wavelength-routed optical networks," IEEE Journal of Selected Areas in Communications vol. 14, no. 5, pp. 840-851, June 1996.

[4] R. Dutta and G. N. Rouskas, "A Survey of Virtual Topology Design Algorithms for Wavelength Routed Optical Networks," Opt. Networks Mag., vol. 1, no. 1, pp. 73-89, Jan. 2000.

[5] B. Mukherjee, S. Ramamurthy, D. Banerjee and A. Mukherjee, "Some principles for designing a wide-area optical network," IEEE/ACM Transactions on Networking vol. 4, no. 5, pp. 684-696, 1996.

[6] O. Crochat, and J-Y. L. Boudec, "Design Protection for WDM Optical Networks," IEE Journal of Selected Areas in Communications, vol. 16, no. 7, September 1998.

[7] A. Sen, B. Hao, B. H. Shen and G. H. Lin, "Survivable routing in WDM networks logical ring in arbitrary physical topology", Proceedings of the IEEE International Communication Conference ICC02, New York, June 2002.

[8] H. Lee, H. Choi, S. Subramaniam, and H. A. Choi, "Survival Embedding of Logical Topology in WDM Ring Networks", Proc. Symposium on Photonics, Networking and Computing, The 6th Joint Conference on Information Sciences (JCIS), Duke University, March 2002.
[9] H. Lee, H. Choi, S. Subramaniam, and H. A. Choi, "Survival Logical Topology Design in WDM Optical Ring Networks", Proc. 39th Allerton conference on communications, control, and computing, Univ. of Illinois at Urbana-Champaign, Oct. 2001.

[10] M. Maliosz, and T. Cinkler, "Confi guration of Protected Virtual Private Networks,” DRCN 2001, Budapest, Hungary, Octomber 2001.

[11] F. Giroire, A. Nucci, N. Taft, C. Diot, "Increasing the Robustness of IP Backbones in the Absence of Optical Level Protection," IEEE INFOCOM, San Francisco 2003.

[12] I. P. Kaminow and T. L. Koch, Eds., Optical Fiber Telecommunications IIIA, Academic Press, 1997.

[13] D. B. West, "Introduction to graph theory," Second Edition, Prentice hall, New Jersey, 1996.

[14] M. R. Garey and D. S. Johnson, "Computers and Intractability: A guide to the theory of NP-completeness", W. H. Freeman and Company, New York.

[15] J. Y. Yen, "Finding the $\mathrm{k}$ shortest loop less paths in a network," Management Science vol. 17, pp. 712-716, 1971. 